\title{
DESIGN OPTIMIZATION OF THE PROPOSED ISAC-2 PROJECT AT TRIUMF
}

\author{
R.E. Laxdal and R.A. Baartman
}

\begin{abstract}
A radioactive ion beam facility, ISAC, is presently under construction at TRIUMF. The post-accelerator takes ions of $A / q \leq 30$ from $2 \mathrm{keV} / \mathrm{u}$ to energies up to $1.5 \mathrm{MeV} / \mathrm{u}$. For the next five year plan, it is intended to increase the final energy above the Coulomb barrier (roughly $6.5 \mathrm{MeV} / \mathrm{u}$ ) and broaden the mass range up to roughly $A=150$. The ISAC2 proposal utilizes the existing RFQ, and a new IH drift tube linac to reach a new stripping energy of $0.4 \mathrm{MeV} / \mathrm{u}$. A post-stripper superconducting linac will accelerate ions with $3 \leq A / q \leq 7$ to $6.5 \mathrm{MeV} / \mathrm{u}$ for the heaviest ions and to more than twice this value for the lightest ions. This paper documents the optimization of the ISAC-2 linac design.
\end{abstract}

\section{INTRODUCTION}

The ISAC facility now under construction at TRIUMF[1] will provide exotic ions of mass $A \leq 30$ up to energies of $1.5 \mathrm{MeV} / \mathrm{u}$. The post-accelerator (dashed area in Fig. 2) consists of a $35 \mathrm{MHz}$ RFQ to increase the energy of ions with $A / q \leq 30$ from $2 \mathrm{keV} / \mathrm{u}$ to the $150 \mathrm{keV} / \mathrm{u}$ stripping energy and a post-stripper $105 \mathrm{MHz}$ drift-tube linac (DTL) to accelerate ions with $3 \leq A / q \leq 6$ to a final energy fully variable between 0.15 and $1.5 \mathrm{MeV} / \mathrm{u}$. All linac components operate $\mathrm{cw}$ to maintain the intensity of the ions.

For the next five year funding segment beginning April 2000 it is proposed to upgrade the present ISAC facility. The proposed expansion, ISAC-2, would extend the accepted mass range up to 150 and the final energy to above the Coulomb barrier $(E \geq 6.5 \mathrm{MeV} / \mathrm{u})$ making TRIUMF a unique world class center for exotic beam investigations.

\section{OVERVIEW OF SCHEME}

The ISAC-2 proposal utilizes the existing ISAC-1 RFQ for low energy acceleration and therefore requires that the ion charge from the source obeys $A / q \leq 30$. Recent advances in charge state booster (CSB) development[3] make this choice feasible. A CSB will be added after the massseparator to increase the charge state of ions with $A>30$.

Assuming a single stripping stage the total voltage required to reach $6.5 \mathrm{MeV} / \mathrm{u}$ from $2 \mathrm{keV} / \mathrm{u}$ is given by

$$
V_{\text {tot }}=\frac{A}{q_{i}}\left(E_{s}-0.002\right)+\frac{A}{q_{s}}\left(6.5-E_{s}\right)
$$

where $q_{i}$ is the initial charge injected into the RFQ, $q_{s}$ is the charge state after stripping and $E_{s}$ is the stripping energy. The total voltage required for accelerating several different ions to $6.5 \mathrm{MeV} / \mathrm{u}$ as a function of stripping energy is given in Fig. 1. The optimum energy for stripping is $\sim 400 \mathrm{keV} / \mathrm{u}$ with stripping efficiencies varying from $50 \%$ for the lightest ions to $15 \%$ for $A=150$.

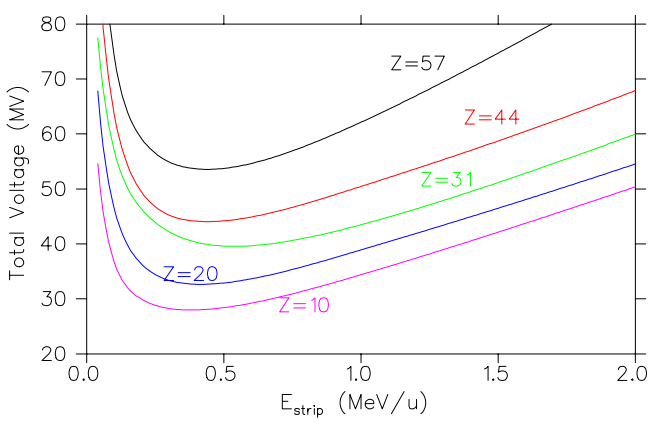

Figure 1: The total voltage required to accelerate various ions from 0.15 to $6.5 \mathrm{MeV} / \mathrm{u}$ as a function of stripping energy.

A room-temperature DTL operating in cw mode will be used to accelerate ions of $A / q \leq 30$ from the RFQ from $0.15-0.4 \mathrm{MeV} / \mathrm{u}$. The beam then is stripped and the ion charge selected in a new MEBT-2 that bends the beam through $90^{\circ}$ to a line parallel to the ISAC-1 DTL line. Ions of mass to charge ratio $3 \leq A / q \leq 7$ are matched into a superconducting DTL on this line and accelerated to at least $6.5 \mathrm{MeV} / \mathrm{u}$ and then transported to the experimental stations. A summary of the linac specifications is shown in Table 1. A schematic of the proposed ISAC-2 linear accelerator complex is shown in Fig. 2.

Table 1: Summary of ISAC-2 linac specifications.

\begin{tabular}{|c|c|c|c|c|}
\hline Device & $\begin{array}{c}E_{\text {in }} \\
(\mathrm{MeV} / \mathrm{u})\end{array}$ & $\begin{array}{c}E_{\text {out }} \\
(\mathrm{MeV} / \mathrm{u})\end{array}$ & $A / q$ & $\begin{array}{c}\Delta V_{\max } \\
(\mathrm{MV})\end{array}$ \\
\hline CSB & - & 0.002 & $\leq 30$ & 0.06 \\
RFQ1 & 0.002 & 0.15 & $\leq 30$ & 4.44 \\
IH-DTL2 & 0.150 & 0.40 & $\leq 30$ & 7.50 \\
strip & & & $3 \rightarrow 7$ & 42.7 \\
SC-DTL & 0.40 & 6.5 & $3 \rightarrow 7$ \\
\hline
\end{tabular}

\section{CHARGE STATE BOOSTER}

The CSB would take the singly charged radioactive beam from the mass-separator and boost the charge state to be compatible with $A / q \leq 30$ for the RFQ. Both ECR and EBIS sources are being considered. An attractive option is to develop a charge state booster which would give $A / q \leq$ 7. This would obviate the need for any stripping and the inherent loss of intensity. Recent results suggest that efficiencies of $2 \%$ are possible in this latter mode and from $10-20 \%$ in the low- $q$ mode[3]. A budget amount of $\mathbf{2} \mathbf{M} \$$ has been allocated for this project. 


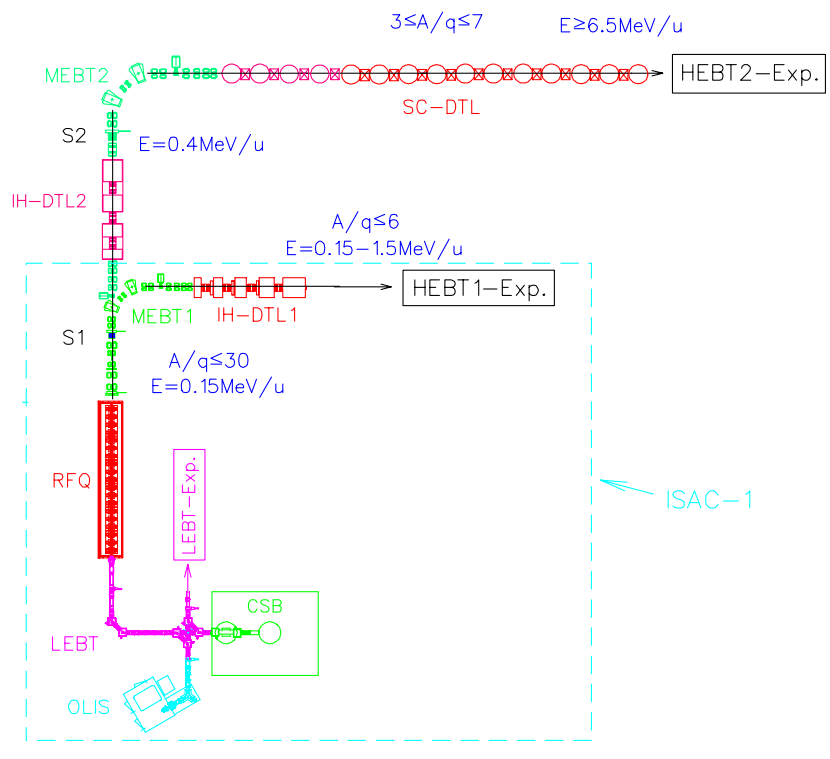

Figure 2: The ISAC-2 linear accelerator complex.

\section{PRE-STRIPPER LINAC}

The IH linac structure operates at very high shunt impedance values making $\mathrm{cw}$ operation at room temperature possible. In ISAC-2 the application is for a fixed final velocity, so long tanks each containing many drift tubes are used to achieve the highest acceleration efficiency. Magnetic quadrupoles are installed both in tanks and between tanks to provide periodic transverse focusing. The small longitudinal and transverse emittances from the RFQ $\left(\epsilon_{z}=0.3 \pi \mathrm{keV} / \mathrm{u} \mathrm{ns}\right.$ and $\left.\beta \epsilon_{x, y}=0.1 \pi \mathrm{mm} \mathrm{mrad}\right)$ allow a frequency of $70 \mathrm{MHz}$, double that of the RFQ frequency, reducing the size of the DTL tanks and improving the shunt impedance. The shunt impedance for the structure is estimated to be $\sim 300 \mathrm{M} \Omega / \mathrm{m}$. In a cw DTL the dissipated power is a more limiting factor than the peak surface field in establishing the operating gradient. A power dissipation of $20 \mathrm{~kW} / \mathrm{m}$ can be safely cooled. The relation $P_{l}=\left(\overline{E_{0} T}\right)^{2} / Z$, where $P_{l}$ is the power per unit length and $Z$ is the effective shunt impedance then gives an average effective gradient $\overline{E_{0} T}$ of $2.4 \mathrm{MV} / \mathrm{m}$.

A schematic diagram of the linac is shown in Fig. 3. In order to reduce the demands on the rf amplifier the DTL is divided into two tanks each with one quadrupole triplet inside roughly midway down the tank and one quadrupole triplet between tanks. Each triplet is $\sim 65 \mathrm{~cm}$ long with gradients up to $60 \mathrm{~T} / \mathrm{m}$. A diagnostic box will also be added to the intertank region. The total length of the linac is $5.8 \mathrm{~m}$. The beam dynamics utilizes the method developed at GSI[2] where a short $-60^{\circ}$ section is used after each magnet system for rebunching followed by an accelerating section at a synchronous phase of $0^{\circ}$. A summary of the tank specifications is given in Table 2. The acceptance of the linac is $\epsilon_{z}=3.6 \pi \mathrm{keV} / \mathrm{u}$ ns and $\beta \epsilon_{x, y}=0.8 \pi \mathrm{mm} \mathrm{mrad}$. With the expected beams from the RFQ the emittance increase during acceleration is calculated to be less than $5 \%$.
Table 2: Specifications for the pre-stripper IH-DTL.

\begin{tabular}{|c|c|c|}
\hline Parameter & Tank 1 & Tank 2 \\
\hline Energy Range & $150-250 \mathrm{keV} / \mathrm{u}$ & $250-400 \mathrm{keV} / \mathrm{u}$ \\
$A / q$ & 30 & 30 \\
$V_{\text {eff }}$ & $3.0 \mathrm{MV}$ & $4.5 \mathrm{MV}$ \\
No. of cells & 13,21 & 21,20 \\
$E_{g}(\mathrm{MV} / \mathrm{m})$ & 2.7 & 2.7 \\
Length $(\mathrm{m})$ & 2.2 & 3.0 \\
Power $(\mathrm{kW})$ & 30 & 45 \\
\hline
\end{tabular}

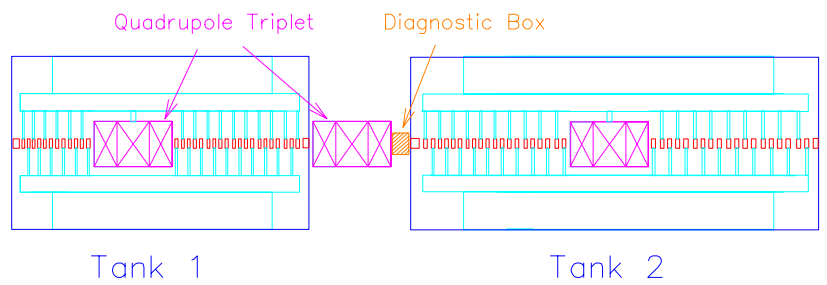

Figure 3: The pre-stripper IH-linac for ISAC-2.

\section{POST-STRIPPER LINAC}

Recent improvements in accelerating gradient and simplification of fabrication procedures[4] plus flexible operation and high beam quality make a superconducting linac a favourable choice for the ISAC-2 post-stripper accelerator. Superconducting heavy ion linacs are composed of several independently fed cavities arranged in a common cryostat with focusing magnets at periodic intervals down the length. In the ISAC-2 linac we choose a geometry having four resonators in a cylindrical vertically mounted cryostat with room-temperature quadrupoles between cryostats. A two-gap quarter wave structure was chosen for its high velocity acceptance and inherent mechanical stability. The former is useful to efficiently accelerate the wide range of ions with a minimum of cavity types and the latter is essential to produce high accelerating gradients.

For optimization the acceleration efficiency is calculated for both two- and three-gap cavities each using two or three different $\beta$ regimes. Efficiencies over the whole $A / q$ range are calculated assuming a fixed acceleration gradient. The conclusion is that linac performance even with a two- $\beta$, two-gap solution is remarkably good, with an integrated time constant of better than $82 \%$ over the whole mass range compared to a theoretical maximum time constant of $90 \%$. The acceleration efficiency for $A / q=7$ and $A / q=3$ for two-gap cavities with two $\beta$ sections (SC-DTL1 and SCDTL2) corresponding to $\beta_{0}$ values of $4.8 \%$ and $9.6 \%$ and a voltage gain ratio in respective sections of 1:3 are shown in Fig. 4. To reach $V_{\text {eff }}=42.7 \mathrm{MV}$ with an integrated time constant of $82 \%$ and an average synchronous phase of $-25^{\circ}$ requires a voltage of 57.5 MV with roughly $14.4 \mathrm{MV}$ and 43.1 MV from the low- and high- $\beta$ sections respectively. 


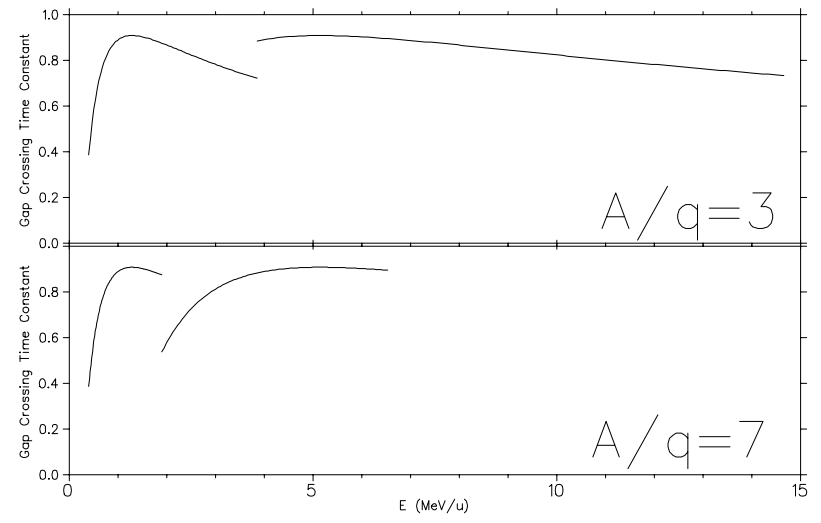

Figure 4: The acceleration efficiency for $A / q=7$ and $A / q=3$ for two-gap cavities with low- and high- $\beta$ sections corresponding to $\beta_{0}$ values of $4.8 \%$ and $9.6 \%$.

Cavity Dimensions Once the design $\beta_{0}$ is established the cavity dimensions are set by the rf frequency choice. The beam is bunched at $11.7 \mathrm{MHz}$ and therefore many harmonics are available. A lower frequency increases the cavity length, hence reduces the required number of cavities but requires a longer inner conductor where mechanical oscillations may be problematic. For the present example we choose frequencies of $70 \mathrm{MHz}$ and $140 \mathrm{MHz}$, the $6^{\text {th }}$ and $12^{\text {th }}$ harmonics of the bunch frequency, for the lowand high- $\beta$ linac sections respectively. (The beam emittance is small enough and the inherent acceptance of the SC-linac large enough that higher frequencies are possible if required.) This gives a fixed cavity diameter of $20.5 \mathrm{~cm}$, hence a common cryostat diameter for both sections of about $1 \mathrm{~m}$, and cavity heights of $107 \mathrm{~cm}$ and $53.5 \mathrm{~cm}$.

Linac Structure Recent developments at Legnaro[4] have shown that cavities of bulk Niobium or with Niobium films sputtered on a copper substrate can deliver gradients consistently above $5 \mathrm{MV} / \mathrm{m}$ and in some cases as high as $8 \mathrm{MV} / \mathrm{m}$ with cooling loads of $7 \mathrm{~W}$ at $4^{\circ} \mathrm{K}$. We have decided to set the number of cavities based on a gradient of $5 \mathrm{MV} / \mathrm{m}$ with a lattice that is compatible with gradients up to $10 \mathrm{MV} / \mathrm{m}$. Each cavity is then capable of an accelerating voltage of $1 \mathrm{MV}$. We opt for 16 low- $\beta$ cavities ( 4 cryostats) and 44 high- $\beta$ cavities (11 cryostats) for $60 \mathrm{MV}$. (Note that this is somewhat different than the optimum values quoted above since cavities are installed in units of four.) Due to the strength of the accelerating fields and hence the rf defocusing, it is proposed to use quadrupole triplets between cryostats to refocus the beam into the next cryostat. Even in this case accelerating gradients are limited to $5 \mathrm{MV} / \mathrm{m}$ and $7.5 \mathrm{MV} / \mathrm{m}$ in the first two cryo-stats while $10 \mathrm{MV} / \mathrm{m}$ can be tolerated in the remainder of the linac. It is also possible to provide a four-quadrupole transition cell after the low energy section and change the lattice to a triplet every second cryostat at the expense of a larger average beam size. The transverse focusing sections are $50 \mathrm{~cm}$ long for the low- $\beta$ section and $60 \mathrm{~cm}$ long in the high- $\beta$ section with maxi- mum gradients of $70 \mathrm{~T} / \mathrm{m}$. A short diagnostic box will be included in each inter-cryostat region. A summary of the specifications for the post-stripper linac is given in Table 3.

Table 3: Specifications for each section of the post-stripper superconducting DTL.

\begin{tabular}{|c|c|c|}
\hline Parameter & SC-DTL1 & SC-DTL2 \\
\hline$E(A / q=7)$ & $0.4-2 \mathrm{MeV} / \mathrm{u}$ & $2-7 \mathrm{MeV} / \mathrm{u}$ \\
$E(A / q=3)$ & $0.4-4.2 \mathrm{MeV} / \mathrm{u}$ & $4.2-15 \mathrm{MeV} / \mathrm{u}$ \\
$A / q$ & $3-7$ & $3-7$ \\
$\beta_{o}$ & 4.8 & 9.6 \\
$f(\mathrm{MHz})$ & 70 & 140 \\
$N_{\text {cav }}$ & 16 & 44 \\
$N_{\text {cryo }}$ & 4 & 11 \\
$E_{g}(\mathrm{MV} / \mathrm{m})$ & 5 & 5 \\
$V_{o}, V_{\text {eff }}(\mathrm{MV})$ & $16,11.5$ & $44,33.1$ \\
Length $(\mathrm{m})$ & 6.0 & 18.5 \\
\hline
\end{tabular}

Beam Simulations Beams of initial emittance $\beta \epsilon_{x, y}=$ $0.2 \pi \mathrm{mm}-\mathrm{mrad}$ and $1.6 \pi \mathrm{keV} / \mathrm{u}-\mathrm{ns}$ and for $A / q=3$ and $A / q=7$ were simulated in the linac code LANA[5] using a triplet every cryostat and a gradient of $5 \mathrm{MV} / \mathrm{m}$. Final beam energies were $14.9 \mathrm{MeV} / \mathrm{u}$ and $6.9 \mathrm{MeV} / \mathrm{u}$ respectively. Growth in the transverse and longitudinal emittance was less than $5 \%$. The longitudinal acceptance in both cases was $50 \pi \mathrm{keV}$-ns; 30 times larger than the design input beam.

\section{COSTS AND SCHEDULE}

The estimated cost of the upgrade, not including internal manpower or building expansion, is $18 \mathrm{M} \$$ (Can). The proposal is now before the funding agency. A decision on the project is expected by Feb. 2000. Assuming the requested funds are obtained, first beams at $5 \mathrm{MV} / \mathrm{m}$ and at a limited mass range would be available as early as 2003 with the complete facility operational in April 2005.

\section{REFERENCES}

[1] P. Schmor, "Initial Commissioning of the ISAC Radioactive Beam Facility", these proceedings.

[2] U. Ratzinger, "A Low Beta RF Linac Structure of the IH type with Improved Radial Acceptance", Proceedings of 1988 Linear Accel. Conf., Newport News Virginia, June 1989.

[3] R. Geller, "Development of ECR Plasmas for Radioactive Ion Beams", Proceedings of HIAT98, Argonne Nat. Lab. 1998, to be published.

[4] A. Facco, et al, "Superconducting Cavity Development at Legnaro", Proceedings of HIAT98, Argonne Nat. Lab. 1998, to be published.

[5] D.V. Gorelov and P.N. Ostroumov, "The LANA Computer Code for the Beam Dynamics Simulation in Multi-Cavity Linacs”, INR Internal Report, 1993-1994. 